\title{
Publisher Correction: The lipid code-dependent phosphoswitch PDK1-D6PK activates PIN-mediated auxin efflux in Arabidopsis
}

Shutang Tan, Xixi Zhang, Wei Kong, Xiao-Li Yang, Gergely Molnár, Zuzana Vondráková, Roberta Filepová, Jan Petrášek, Jiří Friml and Hong-Wei Xue

Correction to: Nature Plants https://doi.org/10.1038/s41477-020-0648-9, published online 11 May 2019.

In the HTML version of this Article originally published, Hong-Wei Xue was mistakenly not designated as a corresponding author; this has now been corrected. The PDF version was unaffected.

Published online: 12 June 2020

https://doi.org/10.1038/s41477-020-0719-y

๑ The Author(s), under exclusive licence to Springer Nature Limited 2020 